\title{
Equilibria between the $\mathrm{K}^{+}$binding and cation vacancy conformations of potassium channels
}

\section{Dear Editor,}

Potassium channels are integral membrane proteins that selectively conduct $\mathrm{K}^{+}$ions across cell membrane (Hille, 2001). They play essential roles in maintaining cellular ionic homoeostasis and generating action membrane potentials in excitable cells. The mechanism of $\mathrm{K}^{+}$selectivity has been evaluated in many biophysical and physiological studies (Zhou et al., 2001; Liu et al., 2015; Schewe et al., 2016). A highly conserved signature sequence, TVGYG, in the selectivity filter of potassium channels (e.g., KcsA) is known to coordinate $\mathrm{K}^{+}$ions (Zhou et al., 2001). Carbonyls of these residues point toward the pore, forming four continuous ion binding sites (S1-S4) and resulting in higher selectivity for $\mathrm{K}^{+}$over $\mathrm{Na}^{+}$(Zhou et al., 2001). The NaK channel from Bacillus cereus is a non-selective cation channel that shares high structural homology with KcsA (Shi et al., 2006). Owing to a distinctive primary sequence of ${ }_{63} T_{V G D G}$, the selectivity filter of NaK preserves only two ion binding sites, allocated similarly as S3 and S4 in KcsA (Alam and Jiang, 2009a, b). Remarkably, the D66Y and N68D double mutations of NaK channel (Fig. S1) transform it into a $\mathrm{K}^{+}$selective channel (termed as NaK2K) (Sauer et al., 2013). Crystal structure of NaK and NaK2K have revealed distinct binding coordination of $\mathrm{Na}^{+}$and $\mathrm{K}^{+}$ions in their selectivity filter (Figs. S2 and S3) (Alam and Jiang, 2009a, b; Sauer et al., 2013). However, dynamics of the NaK and NaK2K selectivity filter with the binding of $\mathrm{Na}^{+}$or $\mathrm{K}^{+}$are still elusive. Especially, it has been known that the membrane environment is highly diverse from detergent micelles, which was considered to influence the structure and function of membrane proteins dramatically (Cross et al., 2011). Thus, it is necessary to study the cation and binding properties of NaK NaK2K channels in lipid bilayers.

Solid-state NMR (ssNMR) is frequently applied to study the structure and dynamics of membrane proteins in lipid bilayers (Lange et al., 2006; Hu et al., 2010). Chemical shift and line shape of ssNMR resonance are sensitive to local variations surrounding the detection nuclei. Therefore, the ${ }^{17} \mathrm{O}$ or ${ }^{13} \mathrm{C}$ isotope labelled carbonyl groups in the selectivity filter of NaK and NaK2K can act as perfect NMR probes to reflect subtle environmental variations, including nearby ions binding as well as the dynamics of the selectivity filter themselves. Nevertheless, it is rather difficult to acquire ${ }^{17} \mathrm{O}$ NMR signals, simply because of the difficulties in sitespecific isotope labelling and the complicated date interpretation of quadruple ${ }^{17} \mathrm{O}$ spins (Wu 2016). In this work, site-specific Val64 $-{ }^{13} \mathrm{C}(\mathrm{O})$ resonances of $\mathrm{NaK}$ and NaK2K at different cation conditions were acquired to provide insight into the ion binding process for both non-selective NaK channel and $\mathrm{K}^{+}$selective NaK2K channel.

Considering there is only a single pair of Valine-Glycine dipeptide pair along the primary sequence of $\mathrm{NaK}$ or NaK2K (Fig. $1 \mathrm{~A}$ ), the site-specific Val64- ${ }^{13} \mathrm{C}(\mathrm{O})$ signals could be obtained through combination of two amino acids $\left({ }^{13} \mathrm{C}(\mathrm{O})\right.$ Val and ${ }^{15} \mathrm{~N}$-Gly) specific labelling in NaK or NaK2K samples, and using NCO double cross polarization (DCP) ssNMR pulse sequences (Fig. 1C). The amino acids labelled NaK and NaK2K were expressed in M9 medium, purified in the $n$-decyl- $\beta$-D-maltoside (DM), and reconstituted into DMPC/DMPG (3:1) lipid bilayers. The tetrameric NaK and NaK2K channel were still maintained in proteoliposomes, as shown by SDS-PAGE (Fig. S4B). Single-channel recordings verified the well-folded and functional NaK channel in lipids (Fig. S4C). The ssNMR experiments were performed on a $600 \mathrm{MHz}$ wide-bore spectrometer. Using the NCO DCP pulse sequence (Fig. 1C), magnetization of the ${ }^{15} \mathrm{~N}$-Gly was initially built up through cross polarization transfer from ambient protons, then transferred to a directly bonded ${ }^{13} \mathrm{C}$ (O) spin, thus resulting in high specificity for the dipeptide ${ }^{13} \mathrm{C}(\mathrm{O})-$ Val64- ${ }^{15} \mathrm{~N}-\mathrm{Gly} 65$ (Fig. 1B). The NCO DCP also effectively suppressed the natural abundance ${ }^{13} \mathrm{C}$ signals, especially from lipids (Figs. 1D, S6 and S7).

First, we collected one-dimensional NCO spectra of $\left[{ }^{15} \mathrm{~N}\right.$ Gly, ${ }^{13} \mathrm{CO}$-Val]-labelled NaK or NaK2K in lipid bilayers without any metal cation and obtained $\mathrm{S}_{0}$ resonances for $\mathrm{NaK}$ and NaK2K, respectively (Fig. $1 \mathrm{E}$ and $1 \mathrm{H}$ ). Excluding the binding of $\mathrm{Na}^{+}$or $\mathrm{K}^{+}$in both samples, the $\mathrm{S}_{0}$ resonances of $\mathrm{NaK}$ and NaK2K could represent specific cation vacancy conformation of $\mathrm{NaK}$ and NaK2K (i.e., without any metal cation coordinated in the selective filter), respectively. The single sharp $S_{0}$ resonance of NaK was observed at 178.51 ppm (Fig. 1E) and line width of this peak was only $0.6 \mathrm{ppm}$ (full-width at half-height, $\mathrm{FWHH}$ ), indicating the vacancy conformation of NaK was rigid and stable. However, the $\mathrm{S}_{0}$ resonance of NaK2K was quite broad (Fig. $1 \mathrm{H})$, indicating a 
A

$$
\begin{aligned}
& \text { 21- DKEFQVLFVLTILTLISGTI -40 } \\
& \text { 41- FYSTVEGLRPIDALYFSVVT -60 } \\
& \text { 61- LTTVEGGNFSPQTDFGKIFT -80 } \\
& \text { 81- ILYIFIGIGLVFGFIHKLAV-100 } \\
& \text { 101- NVQLPSILSN }
\end{aligned}
$$

C
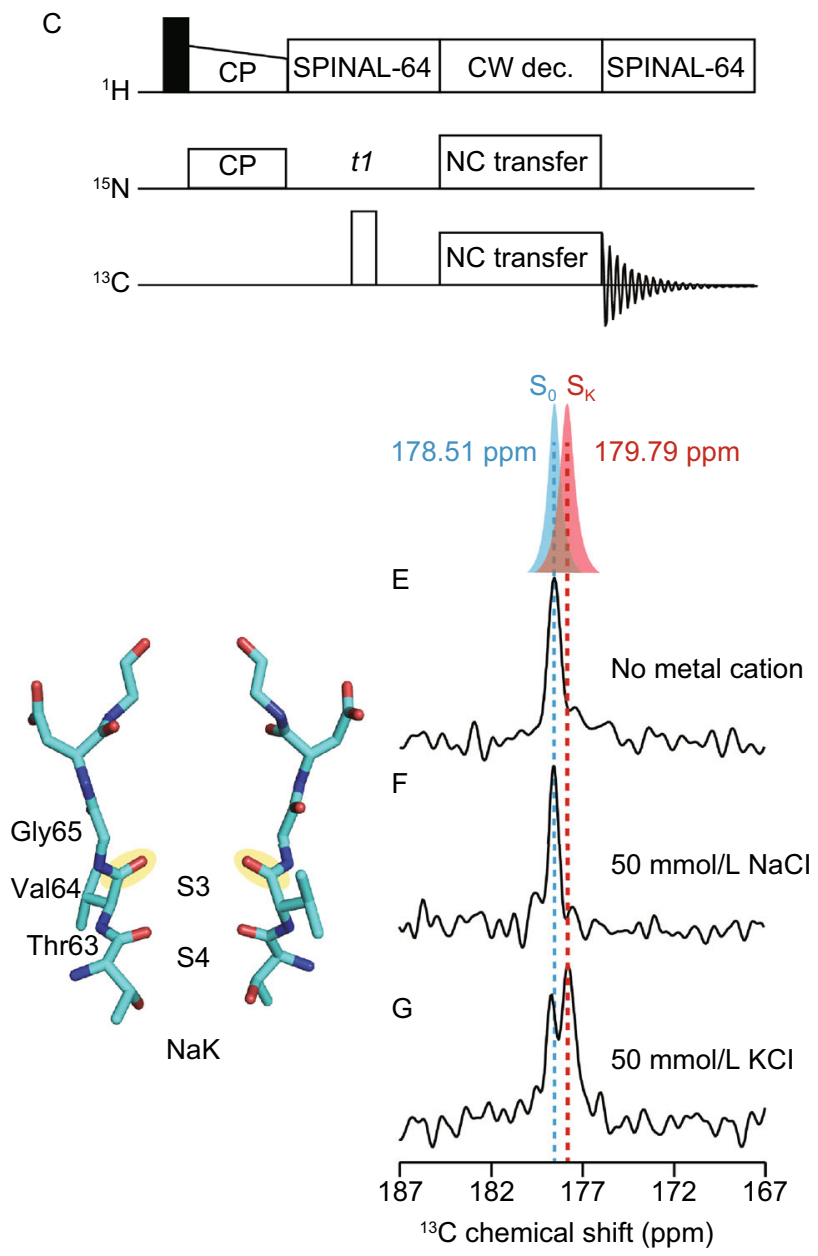

B

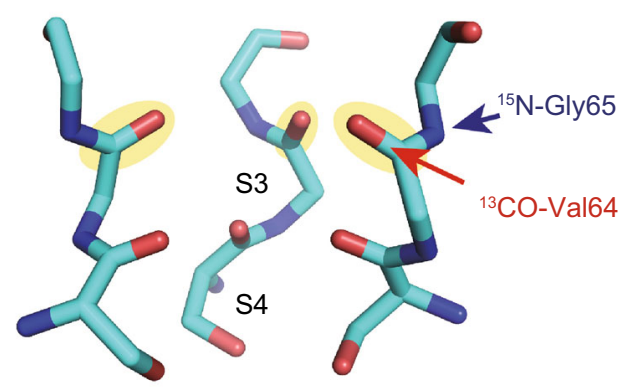

D

$\left[{ }^{15} \mathrm{~N}-\right.$ Gly, ${ }^{13} \mathrm{CO}-$ Val]-labelled NaK

in DMPC/DMPG lipid bilayers

NCO-1D spectrum
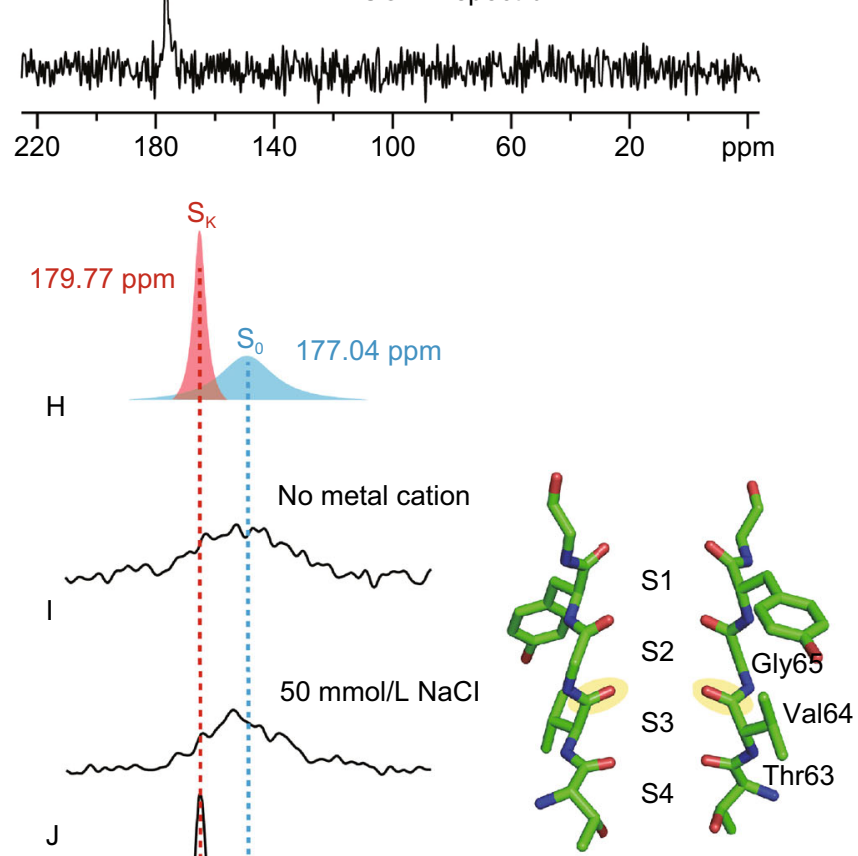

NaK2K

Figure 1. Site-specific isotope labelling strategy and ssNMR analysis of $13 \mathrm{C}(0)$-Val64 resonances at different cation conditions. (A) Amino acid sequence of the NaK channel. Val64 was marked with asterisk. (B) The carbonyl groups of Val64 were shaded in yellow. The front subunit was removed for clarity. (C) Pulse sequence of NCO DCP experiments. Open and filled bars represent $180^{\circ}$ and $90^{\circ}$ pulse, respectively. (D) One-dimensional ${ }^{13} \mathrm{C}-\mathrm{NCO}$ spectrum of $\left[{ }^{15} \mathrm{~N}-\mathrm{Gly},{ }^{13} \mathrm{CO}-\right.$ Val $]$-labelled NaK in DMPC/ DMPG lipid bilayers. $\left[{ }^{15} \mathrm{~N}-\mathrm{Gly},{ }^{13} \mathrm{CO}-\mathrm{Val}\right]$-labelled $\mathrm{NaK}$ in buffer without any metal cation (E), in presence of $50 \mathrm{mmol} / \mathrm{L} \mathrm{NaCl}(\mathrm{F})$ or $50 \mathrm{mmol} / \mathrm{L} \mathrm{KCl}(\mathrm{G}) .\left[{ }^{15} \mathrm{~N}-\mathrm{Gly},{ }^{13} \mathrm{CO}-\mathrm{Val}\right]$-labelled NaK2K in buffer without any metal cation $(\mathrm{H})$, in presence of $50 \mathrm{mmol} / \mathrm{L} \mathrm{NaCl}(\mathrm{I})$ or $50 \mathrm{mmol} / \mathrm{L} \mathrm{KCl}(\mathrm{J})$. The carbonyl groups of Val64 were shaded in yellow on the structure of NaK and NaK2K selectivity filter.

non-homogenous conformation without presence of either $\mathrm{Na}^{+}$or $\mathrm{K}^{+}$.

To further characterize the difference binding properties of $\mathrm{Na}^{+}$and $\mathrm{K}^{+}$in the selectivity filter of NaK or NaK2K, NCO spectra of $\left[{ }^{15} \mathrm{~N}-\mathrm{Gly},{ }^{13} \mathrm{CO}\right.$-Vall-labelled NaK or NaK2K in the presence of $50 \mathrm{mmol} / \mathrm{L} \mathrm{NaCl}$ or $50 \mathrm{mmol} / \mathrm{L} \mathrm{KCl}$ were acquired. As shown in Fig. $1 \mathrm{~F}$ and $1 \mathrm{l}$, the Val64- ${ }^{13} \mathrm{C}(\mathrm{O})$ resonances of $\mathrm{NaK}$ or NaK2K in the presence of $50 \mathrm{mmol} / \mathrm{L}$ $\mathrm{NaCl}$ was quite similar as the $\mathrm{S}_{0}$ resonance of $\mathrm{NaK}$ or NaK2K (Fig. 1E and $1 \mathrm{H}$ ), respectively. Since it is known that 

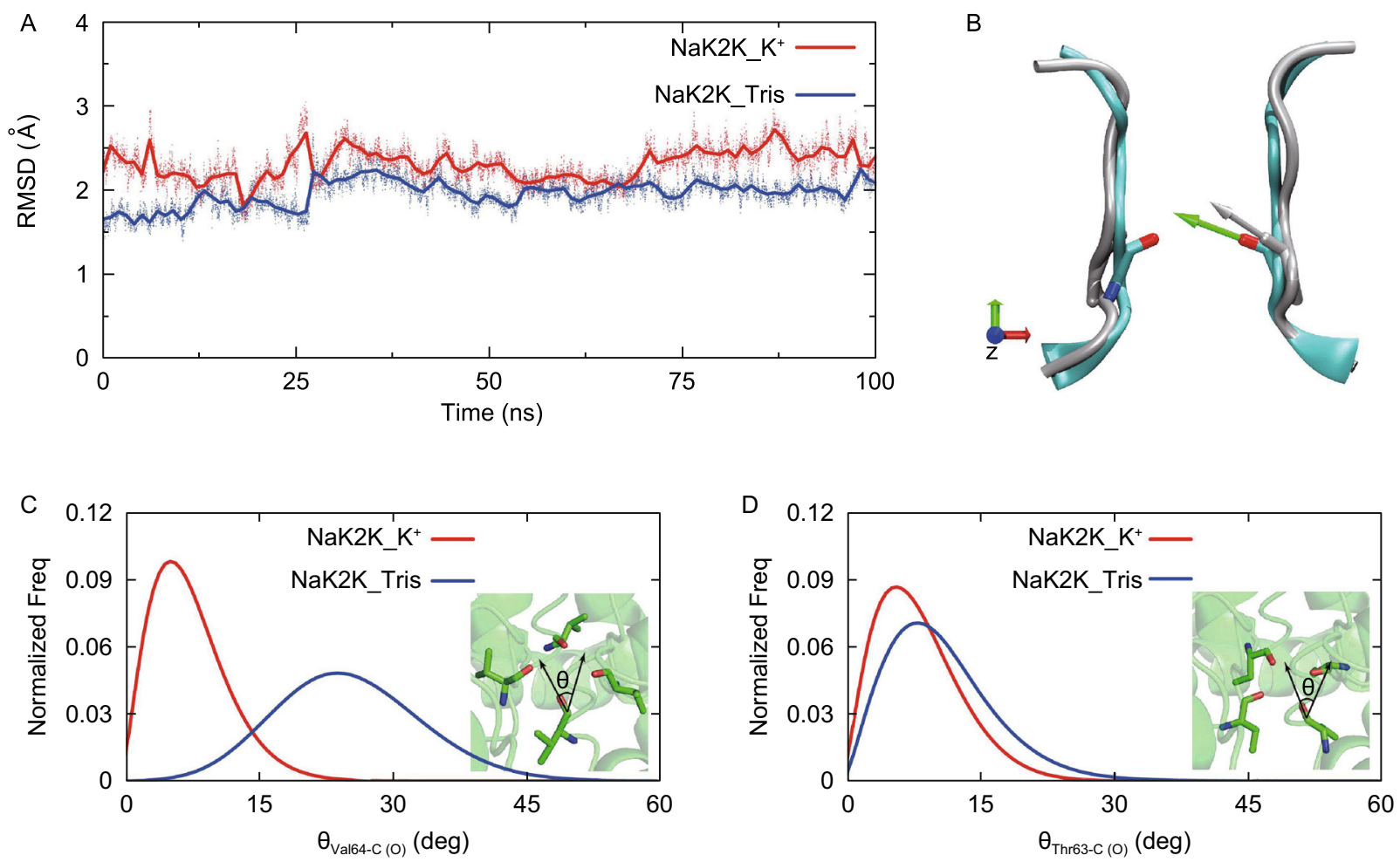

Figure 2. Dynamics of the NaK2K Val64 carbonyl groups during $\mathrm{K}^{+}$binding process. (A) RMSD of the NaK2K_K $\mathrm{K}^{+}$and NaK2K_Tris systems during MD simulation. (B) $\theta_{\text {Val64-C(O) }}$ angle was formed by the two $C=O$ vectors from MD simulations (grey) and the reference crystal structure (cyan). (C) Distribution of $\theta_{\text {Val64-C(O) }}$ in NaK2K_K $K^{+}$system (red) and in NaK2K_Tris system (blue). (D) Distribution of $\theta_{\text {Thr63-C(O) }}$ in NaK2K_K $\mathrm{K}^{+}$system (red) and in NaK2K_Tris system (blue).

the ${ }^{13} \mathrm{C}$ resonances are sensitive to local environment $(\mathrm{Hu}$ et al., 2010), the almost unchanged site-specific Val64- ${ }^{13} \mathrm{C}$ (O) spectra indicated that there must be no $\mathrm{Na}^{+}$binding around the Val64 carbonyl groups of either NaK or NaK2K. This was contradictory to the observed electron density at S3 site in the crystal structure of $\mathrm{NaK}_{-} \mathrm{Na}^{+}$complex (PDB number 3e89) (Alam and Jiang, 2009a, b). Nevertheless, authors of the crystallography studies have mentioned that it was difficult to distinguish the electron densities between $\mathrm{Na}^{+}$and water molecules (Sauer et al., 2013). The observed electron density at S3 site in the crystallography studies might be generated by a water molecule, instead of a binding $\mathrm{Na}^{+}$ion, or a non-specific $\mathrm{Na}^{+}$ion trapped in the low temperature $(\sim 100 \mathrm{~K})$ condition during $\mathrm{X}$-ray diffraction (Fowler et al., 2008).

In the NCO spectra of $\left[{ }^{15} \mathrm{~N}-\mathrm{Gly},{ }^{13} \mathrm{CO}-\right.$ Val]-labelled NaK or NaK2K in the presence of $50 \mathrm{mmol} / \mathrm{L} \mathrm{KCl}$ (Fig. $1 \mathrm{G}$ and $1 \mathrm{~J}$ ), two resonances were observed for each sample. One resonance was similar as $S_{0}$, while the other resonance could be assigned as $S_{k}$-the Val64- ${ }^{13} \mathrm{C}(\mathrm{O})$ resonance of NaK or NaK2K under the influence of $\mathrm{K}^{+}$. Chemical shifts of the well- resolved $S_{K}$ resonances were $177.79 \mathrm{ppm}$ and $179.77 \mathrm{ppm}$ for NaK and NaK2K respectively (Fig. $1 \mathrm{G}$ and $1 \mathrm{~F}$ ). The presence of $S_{k}$ strongly indicated the binding of $\mathrm{K}^{+}$in the selectivity filter of NaK and NaK2K. Meanwhile, observation of both $S_{0}$ and $S_{K}$ implied that the cation vacancy conformation $\left(\mathrm{S}_{0}\right.$ resonance) and $\mathrm{K}^{+}$binding conformation $\left(\mathrm{S}_{\mathrm{K}}\right.$ resonance) existed simultaneously in the selectivity filter of $\mathrm{NaK}$ or NaK2K channels. Furthermore, similar integrated area of the $S_{0}$ and $S_{k}$ resonances indicated almost equal proportions of the two conformations. These results might suggest the $\mathrm{K}^{+}$flux coming through $\left(\mathrm{S}_{\mathrm{k}}\right)$ or away from $\left(\mathrm{S}_{0}\right)$ the Val64-C(O) site, which was consistent with the proposed $\mathrm{K}^{+}$selectivity and flux model in KcsA (Morais-Cabral et al., 2001).

As shown in Fig. $1 \mathrm{H}$ and $1 \mathrm{~J}$, the line shape of $\mathrm{S}_{\mathrm{K}}$ and $\mathrm{S}_{0}$ resonances were quite different for $\mathrm{NaK} 2 \mathrm{~K}$, suggesting pronounced dynamics variations of the NaK2K Val64 backbone carbonyl during $\mathrm{K}^{+}$binding process. May due to the structure difference in the hydrogen bond network between pore helix and selectivity filter, NaK2K vacancy conformation shows a slower dynamic in the filter than the KcsA C-type 
inactivation conformation, which shows a set of sharp peaks on solid state NMR (Bhate et al., 2010). To validate this possibility, molecular dynamics (MD) simulations were conducted with the duration of $\sim 100 \mathrm{~ns}$ for both NaK2K_Tris (as $\mathrm{S}_{0}$ resonance) and NaK2K_K $\mathrm{K}^{+}$(as $\mathrm{S}_{\mathrm{K}}$ resonance) systems (Fig. 2A). Swing motion of the Val64 carbonyl groups was characterized with the $\theta_{\mathrm{Val64-C(O)}}$ angle, which was formed by the two $C=O$ vectors from $M D$ simulation and the reference crystal structure (Fig. 2B). The optimal $\theta_{\text {Val64-C(O) }}$ angle in the NaK2K_K $K^{+}$system was $5^{\circ}$, while that angle in the NaK2K_Tris system was $24^{\circ}$ (Fig. 2C), indicating different Val64- $\mathrm{C}(\mathrm{O})$ orientation with respect to the normal direction of the channel pore. Meanwhile, the angle distribution of $\theta_{\text {Val64-C(O) }}$ in the NaK2K_K $K^{+}$system $\left(0^{\circ}\right.$ to $\left.20^{\circ}\right)$ were much narrower than that of NaK2K_Tris system $\left(5^{\circ}\right.$ to $\left.50^{\circ}\right)$, demonstrating the more stable and rigid conformation of Val64-C(O) upon the binding of $\mathrm{K}^{+}$(Fig. 2C). As references, the angle distribution of $\theta_{\text {Thr63-C(O) }}$ in both systems were also analyzed and only minor variations were observed (Fig. 2D). The correlation analysis of $\theta_{\mathrm{Val64}-\mathrm{C}(\mathrm{O})}$ and $\theta_{\text {Thr63-C(O) }}$ angels in either NaK2K_K ${ }^{+}$(Fig. S8A) or NaK2K_Tris (Fig. S8b) system also suggested the high sensitivity of Val64-C (O) than Thr63-C(O) during $\mathrm{K}^{+}$binding in the selectivity filter of NaK2K. Overall, our SSNMR line shape analysis and parallel MD simulation data highly verified the previous hypothesis that the binding of $\mathrm{K}^{+}$ions could stabilize the selectivity filter of NaK2K, which might result in the "conducting" state.

In summary, we have obtained the site-specific Val64- ${ }^{13} \mathrm{C}$ (O) spectra of NaK and NaK2K in lipid bilayers through the combination of double amino acid specific labeling and NCO DCP ssNMR experiments. The analysis of Val64- ${ }^{13} \mathrm{C}$ (O) resonances at different $\mathrm{Na}^{+} / \mathrm{K}^{+}$conditions indicated no $\mathrm{Na}^{+}$ion binding in the vicinity of NaK-Val64-C(O) and suggested that both $\mathrm{K}^{+}$-selective NaK2K channel and non-selective NaK channel preferred to bind $\mathrm{K}^{+}$ions in the central part of the selectivity filter. We also observed the distinctive equilibria between the $\mathrm{K}^{+}$binding conformation and cation vacancy conformation in selectivity filter of NaK or NaK2K through ssNMR methods. The experimental line-width analysis and $M D$ simulations verified the conformational stabilization of NaK2K selective filters upon the binding of $\mathrm{K}^{+}$ ions. The two conformations might show in ion channels simultaneously. The $\mathrm{S}_{\mathrm{k}}$ conformation provide the $\mathrm{K}^{+}$conduction pathway which dehydrated $\mathrm{K}^{+}$can pass through in single file. The $S_{0}$ conformation may conduct partially hydrated $\mathrm{Na}^{+}$(Kuhlbrandt, 2016), which leads a similar chemical environment for V64 in the vacancy conformation with water molecules. Methods in this study could be applied to shed more insights into the ion binding process of ion channels in native-like membrane environments and to illustrate physical chemistry mechanisms of other tetrameric ion channels.

\section{FOOTNOTES}

This work was supported by the Chinese Key Research PlanProtein Science (2015CB910100 and 2016YFA0400900), and the Chinese Natural Science Foundation (U1332138 and U1432136), the CAS-Hefei Science Center grant (2015HSC-SRG051).

The authors declare that there is no conflict of interest.

This article does not contain any studies with human or animal subjects performed by the any of the authors.

Yao He ${ }^{1}$, Bo Zhang ${ }^{1}$, Hao Dong ${ }^{2 \bowtie}$, Penglin $\mathrm{Xu}^{3}$, Xiaoying Cai ${ }^{1}$, Ting Zhou ${ }^{3}, \mathrm{Mu} \mathrm{Yu}^{1}$, Jun Liang ${ }^{1}$, Xiao Zheng ${ }^{3 凶}$, Changlin Tian ${ }^{1 凶}$

${ }^{1}$ Hefei National Laboratory for Physical Sciences at the Microscale and School of Life Sciences, University of Science and Technology of China, Hefei 230027, China

${ }^{2}$ Kuang Yaming Honors School and Institute for Brain Sciences, Nanjing University, Nanjing 210023, China

${ }^{3}$ Hefei National Laboratory for Physical Sciences at the Microscale and School of Chemistry and Material Sciences, University of Science and Technology of China, Hefei 230026, China

$\triangle$ Correspondence: donghao@nju.edu.cn (H. Dong), xz58@ustc.edu.cn (X. Zheng), cltian@ustc.edu.cn (C. Tian)

\section{OPEN ACCESS}

This article is distributed under the terms of the Creative Commons Attribution 4.0 International License (http://creativecommons.org/ licenses/by/4.0/), which permits unrestricted use, distribution, and reproduction in any medium, provided you give appropriate credit to the original author(s) and the source, provide a link to the Creative Commons license, and indicate if changes were made.

\section{REFERENCES}

Alam A, Jiang Y (2009a) High-resolution structure of the open NaK channel. Nat Struct Mol Biol 16:30-34

Alam A, Jiang $Y(2009 b)$ Structural analysis of ion selectivity in the NaK channel. Nat Struct Mol Biol 16:35-41

Bhate MP, Wylie BJ, Tian L, McDermott AE (2010) Conformational dynamics in the selectivity filter of KcsA in response to potassium ion concentration. J Mol Biol 401:155-166

Cross TA, Sharma M, Yi M, Zhou HX (2011) Influence of solubilizing environments on membrane protein structures. Trends Biochem Sci 36:117-125

Fowler PW, Tai K, Sansom MS (2008) The selectivity of $\mathrm{K}^{+}$ion channels: testing the hypotheses. Biophys J 95:5062-5072

Hille B (2001) lon channels of excitable membranes. Sinauer, Sunderland, MA 
Hu F, Luo W, Hong M (2010) Mechanisms of proton conduction and gating in influenza M2 proton channels from solid-state NMR. Science 330:505-508

Kuhlbrandt W (2016) Three in a row-how sodium ions cross the channel. EMBO J 35:793-795

Lange A, Giller K, Hornig S, Martin-Eauclaire MF, Pongs O, Becker S, Baldus M (2006) Toxin-induced conformational changes in a potassium channel revealed by solid-state NMR. Nature 440:959-962

Liu S, Lv P, Li D, Guo X, Zhang B, Yu M, Li D, Xiong Y, Zhang L, Tian $C(2015) K^{+}$preference at the NaK channel entrance revealed by fluorescence lifetime and anisotropy analysis of site-specifically incorporated (7-hydroxycoumarin-4-yl) ethylglycine. Chem Commun 51:15971-15974

Morais-Cabral JH, Zhou Y, MacKinnon R (2001) Energetic optimization of ion conduction rate by the $\mathrm{K}^{+}$selectivity filter. Nature 414:37-42
Sauer DB, Zeng W, Canty J, Lam Y, Jiang Y (2013) Sodium and potassium competition in potassium-selective and non-selective channels. Nat Commun 4:2721

Schewe M, Nematian-Ardestani E, Sun H, Musinszki M, Cordeiro S, Bucci G, de Groot BL, Tucker SJ, Rapedius M, Baukrowitz T (2016) A non-canonical voltage-sensing mechanism controls gating in $\mathrm{K}_{2} \mathrm{P} \mathrm{K}^{+}$channels. Cell 164:937-949

Shi N, Ye S, Alam A, Chen L, Jiang Y (2006) Atomic structure of a $\mathrm{Na}^{+}$-and $\mathrm{K}^{+}$-conducting channel. Nature 440:570-574

Wu G (2016) Solid-state ${ }^{17}$ O NMR studies of organic and biological molecules: recent advances and future directions. Solid State Nucl Magn Reson 73:1-14

Zhou Y, Morais-Cabral JH, Kaufman A, MacKinnon R (2001) Chemistry of ion coordination and hydration revealed by a $\mathrm{K}^{+}$ channel-Fab complex at $2.0 \AA$ resolution. Nature 414:43-48
Yao He and Bo Zhang contributed equally to this work.

Electronic supplementary material The online version of this article (https://doi.org/10.1007/s13238-019-0609-0) contains supplementary material, which is available to authorized users. 